\title{
OS PRIMÓRDIOS DA ASSISTÊNCIA AOS RECÉM-NASCIDOS NO EXTERIOR E NO BRASIL: PERSPECTIVAS PARA O SABER DE ENFERMAGEM NA NEONATOLOGIA (1870- 1903). ${ }^{1}$
}

\author{
THE EARLY DAYS OF THE ASSISTANCE TO THE NEW-BORN BABIES IN EXTERIOR AND IN BRAZIL: \\ PERSPECTIVES TO KNOWLEDGE IT OF NURSING IN THE NEONATOLOGY (1870-1903). ${ }^{1}$
}

\author{
LOS PRIMÓRDIOS DE LA AYUDA A LOS BEBÉS Recién nacidos EN EXTERIOR Y EN EL BRASIL: \\ PERSPECTIVAS AL CONOCIMIENTO DEL CUIDADO EN NEONATOLOGÍa (1870-1903). ${ }^{1}$
}

\section{Renata Gomes Rodrigues ${ }^{2}$ Isabel Cristina dos Santos Oliveira ${ }^{3}$}

\begin{abstract}
RESUMO: Trata-se de um estudo histórico que tem como objetivo descrever os primórdios da assistência aos recém-nascidos, no exterior e no Brasil, no período de 1870 a 1903. As fontes primárias são artigos existentes na Fundação Biblioteca Nacional. As fontes secundárias são estudos pertinentes à temática. Com a finalidade de manter os prematuros aquecidos, no final do século XIX, foram criadas as primeiras incubadoras. No Brasil, as incubadoras Lion chegaram no início do século XX (1903). O uso das incubadoras e a especificidade dos cuidados aos recém-nascidos são marcos relevantes para o desenvolvimento da neonatologia.
\end{abstract}

PALAVRAS-CHAVES: Enfermagem; Neonatologia; História da Enfermagem.

SUMMARY: It is a historical study that has as objective to describe the early days of the assistance to the newborn babies in foreign countries and in Brazil, from 1870 to 1903. The primary sources are existing articles in the Foundation National Library. The secondary sources are pertinent studies to the thematic one. With the purpose to keep warm the premature babies, in the end of XIX century were created the first incubator. In Brazil, the "Lion" new-born babies arrived in the beginning of XX century (1903). The use of the new born babies and the peculiarities of the cares to the new-born babies are Importants landmarks for the development of the neonatology.

KEY WORDS: Nursing; Neonatology; Nursing History.

RESUMEN: Esto es un estudio histórico que tiene como objetivo describir los primordios de la ayuda a los prematuros en exterior y en el Brasil, en el período de 1870 a 1903. Las fuentes primarias son artículos existentes en la Fundación Biblioteca Nacional, Archivo Municipal do Rio de Janeiro, Centro de la Documentación y de la Biblioteca Sectorial de Pos grado de la Escuela de Enfermería Anna Nery de la Universidad Federal de Rio de Janeiro. Las fuentes secundarias son estudios pertinentes del temático. Con el propósito de mantener caliente a los prematuros, en el final del siglo XIX fue creado la primera incubadora. En Brasil, las incubadoras "Lion" habían llegado en el principio del siglo XX (1903). El uso de los incubadoras y las particularidades de los cuidados a los prematuros es señales excelentes para el desarrollo de la neonatología.

TERMINOSCLAVES: Enfermeria; Neonatologia; Historia de la Enfermeria.

\footnotetext{
1 Este estudo faz parte do Projeto Integrado de Pesquisa / CNPq intitulado: "A prática da enfermagem nos hospitais pediátricos: a influência da tecnologia nos anos 70", coordenado pela Profa. Dra. Isabel Cristina dos Santos Oliveira.

${ }^{2}$ Aluna do $8^{\circ}$ período do Curso de Graduação em Enfermagem e Obstetrícia da Escola de Enfermagem Anna Nery (EEAN)/ Universidade Federal do Rio de Janeiro (UFRJ). Bolsista de Iniciação Científica/ FAPERJ. E-mail: renatarodrigues2003@hotmail.com.

3 Doutora em Enfermagem. Professor Adjunto do Departamento de Enfermagem Médico-Cirúrgica da EEAN/UFRJ. Pesquisadora do CNPq. Orientadora. e-mail: chabucris@ig.com.br.
} 


\section{INTRODUÇÃO}

A motivação em desenvolver este estudo justifica-se pelo meu interesse em entender a assistência aos recém-nascidos e suas especificidades, influenciada pelos avanços tecnológicos. O estudo tem como objetivo: descrever os primórdios da assistência aos recém-nascidos no exterior e no Brasil, no período de 1870 a 1903. Tratase de uma pesquisa histórica, que segundo VIEIRA et al (1989, p. 11) "(...) seria uma forma de recuperar a ação de diferentes grupos e buscar entender o porquê de um processo tomar um dado rumo e não outro".

Os dados foram obtidos através de fontes primárias escritas e fontes secundárias. As fontes primárias escritas são os artigos existentes na Fundação biblioteca Nacional, Arquivo Municipal do Rio de Janeiro e no acervo do Centro de Documentação e Biblioteca Setorial de PósGraduação da Escola de Enfermagem Anna Nery da Universidade Federal do Rio de Janeiro. As fontes secundárias correspondem aos artigos de periódicos nacionais e internacionais pertinentes à temática, livros referentes à Neonatologia e história do Brasil.

\section{A NEONATOLOGIA NO EXTERIOR E NO BRASIL: MARCO HISTÓRICO}

\section{- No Exterior}

Em meados do século XIX, as crianças eram ignoradas pelos médicos, não existiam instituições que se dedicassem aos cuidados com as crianças, eram altas as taxas de mortalidade infantil, principalmente entre os recém-nascidos prematuros.

Segundo AVERY (1999, p.3):

"Esperava-se que as crianças nascidas prematuramente fossem ao êxito letal, assim como também aquelas crianças nascidas com malformações. Havia um sentimento de que a seleção natural se encarregaria das crianças 'menos adaptadas' à sobrevivência, tal como o sugerido pelo termo 'fracote' atribuído as crianças prematuras".

Diante das altas taxas de mortalidade adicionadas da queda nas taxas de natalidade, criou-se um receio na população européia de despovoamento e vulnerabilidade da defesa nacional, nesta época, surgiu na Europa um movimento pela saúde da criança entre 1870 e 1920, que tinha como objetivo preservar a vida de todas as crianças. Este movimento ficou registrado como um dos primeiros momentos da medicina neonatal. Com todo esse movimento social, o cuidado preventivo passou a ser praticado, maternidades foram ampliadas e incubadoras foram fabricadas (LUSSKY, 1999).

Em 1878, um funcionário do zoológico de Paris desenvolveu a pedido do professor e obstetra parisiense, Stephane Etienne Tarnier, uma incubadora semelhante a uma chocadeira de ovos de galinha. Em 1880, o professor apresentou esta incubadora, que foi instalada na Maternidade de Paris (HARRISON, 1946; SILVADO, 1903). LUSSKY (1999, p.1) destaca que: “(...) esta incubadora fez decrescer a taxa de mortalidade de $66 \%$ para $38 \%$ entre crianças pesando menos que $2000 \mathrm{~g}$ ao nascimento".

A Neonatologia é considerada tendo seu início com o obstetra francês Pierre Budin, que estendeu sua preocupação com os recém-nascidos além das salas de parto. Budin criou um ambulatório de puericultura no Hospital Charité, em Paris, no ano de 1892 (AVERY, 1978, p.1), Budin foi o responsável pelo desenvolvimento dos princípios e métodos que passaram a formar a base da medicina neonatal (LUSSKY, 1999).

No final do século XIX, outras evoluções ocorreram no tratamento obstétrico e neonatal. As incubadoras estavam sendo utilizadas no tratamento de crianças prematuras com sucesso, Martin Coney, aluno de Budin, foi para os Estados Unidos em 1896 sendo considerado o primeiro a oferecer cuidados especializados a crianças prematuras. Em Viena, o médico Carl Credé foi o responsável pela introdução do uso de 
nitrato de prata na profilaxia da ophtalmia neonatorum, e um cirurgião ortopedista inglês, Willian Little, correlacionou o trauma de nascimento com a paralisia cerebral. John Baleantyne, um obstetra de Edimburgo, introduziu o tratamento pré-natal e afirmava que: "(...) doenças maternais tal como a sífilis, tifo, tuberculose e a ingestão de toxinas adversas durante a maternidade afetavam a saúde e o desenvolvimento do feto" (LUSSKY, 1999, p.1-2).

Os avanços médicos e tecnológicos da época propiciaram grandes transformações no cuidado neonatal durante e após o parto. Segundo LUSSKY (1999), as fundações que antes eram designadas para prestar assistência a crianças abandonadas foram modificadas e transformadas em hospitais infantis, e os pediatras assumiram um grande papel no tratamento neonatal. Na primeira década do século $X X$, observa-se um aumento da contribuição dos pediatras para a ciência da medicina neonatal, estudos sobre a alimentação (natural e artificial) e a prematuridade foram realizados, e curvas de crescimento eram utilizadas para estabelecer demandas de energia.

Após uma exibição, em Chicago, de lactentes prematuros em uma unidade de demonstração, organizada por Coney, o pediatra Julius Hess criou um centro para assistência aos lactentes prematuros naquela cidade (AVERY, 1978; DOWNES, 1992). HESS (1951 p.1) destaca que: "Não foi antes de 1922 que uma estação para crianças prematuras de porte foi finalmente estabelecida no Sarah Moris Hospital do Michael Reese Hospital em Chicago".

Ainda, no início do século XX, as taxas de mortalidade entre os recém-nascidos mantiveram-se elevadas, além da prematuridade as infecções hospitalares também eram as responsáveis pela maioria dos óbitos. Neste período iniciou-se uma disputa pela assistência aos recém-nascidos entre obstetras e pediatras. LUSSKY (1999, p.2), ressalta, em 1916, que: "a criança recém-nascida estava em uma terra sem homens (no-mansland) entre a obstetrícia e a pediatria".
O pediatra Julius Hess tornou-se a autoridade americana em relação a prematuridade e através dele o tratamento neonatal entrou na cadeira acadêmica, ele também desenvolveu a incubadora Hess. Os hospitais Sarah Morris e Michael Reese Hospital promoveram avanços em técnicas assépticas, serviços de transporte neonatal e alimentação por via nasal (LUSSKY, 1999).

$\mathrm{Na}$ década de 20 ocorre a consolidação e organização dos avanços tecnológicos. Em 1921, um ato social buscou promover o bem estar materno e infantil, e em 1922, inaugurase a primeira e única unidade para crianças prematuras do Sarah Morris Premature Center, o pediatra Julius Hess publica seu compêndio intitulado "Doenças de crianças prematuras e congênitas" (LUSSKY, 1999).

Outras descobertas científicas eram reveladas, e em 1924, Albrecht Peiper descreveu o desenvolvimento neurológico inicial do lactente prematuro; os avanços nas técnicas também se ampliavam e Alfreed Hart realizou, com sucesso, pela primeira vez a exosangüineotransfusão no recém-nascido com icterícia em 1925 (DOWNES, 1992).

Com os avanços técnico-científicos na neonatologia, reduziram-se as taxas de mortalidade, a infecção hospitalar foi controlada com o isolamento estrito do recém-nascido na maternidade, mas isto ocasionou a separação entre mãe e filho, prejudicando 0 vínculo entre eles e o aleitamento.

O marco nos anos trinta foi a criação do "Box de Oxigênio Hess", desenvolvido, pelo pediatra Julius Hess em 1934 e utilizado para o tratamento de distúrbios respiratórios (LUSSKY, 1999, p.3), Faber e Wilson descreveram a síndrome da angústia respiratória em 1932; Albrecht Peiper descreveu distúrbios pulmonares em 1937; em 1938, Levine e Stetson identificaram a incompatibilidade dos grupos sanguíneos materno-fetais e Charles Chapple elaborou uma incubadora moderna conhecida como "Isollete", no Children's Hospital de Filadélfia (DOWNES, 1992). Observa-se nesse período uma maior atenção dada aos problemas respiratórios. 
recém-nascidos no exterior e no Brasil: perspectivas para o saber de enfermagem na neonatologia (1870-1903). Revista Eletrônica de Enfermagem, v. 06, n. 02, p. 286-291, 2004. Disponível em www.fen.ufg.br

\begin{abstract}
A enfermagem também exerceu um papel fundamental no início do desenvolvimento da neonatologia. Um artigo escrito pelo pediatra Julius Hess nos traz a informação de que os melhores resultados obtidos no cuidado aos recém-nascidos prematuros eram alcançados quando enfermeiras bem treinadas estavam à frente do serviço como supervisoras. Neste período cresceu o incentivo pela especialização da enfermagem para 0 cuidado a recémnascidos prematuros, e observa-se um grande investimento nessa área. No Centro para prematuros de Chicago foi criado um fundo de investimento para o treinamento das enfermeiras na área de neonatologia (HESS, 1951).
\end{abstract}

$\mathrm{Na}$ década de 40, os avanços no armazenamento de sangue e nas pesquisas de patologias proporcionaram o desenvolvimento de novas terapias para enfermidades como a eritroblastose fetal (LUSSKY, 1999). Em 1946, Louis Diamond e colaboradores introduziram uma técnica para a exosanguineotransfusão com um cateter de polietileno com base na canulação da veia umbilical com um cateter de polietileno (DOWNES, 1992).

Dando continuidade aos avanços alcançados na década de 30 para os problemas respiratórios, os anos 50 também foram marcados pelas descobertas relacionadas aos distúrbios do trato respiratório. $\mathrm{Na}$ época não havia meios para a respiração mecânica, e a apnéia infantil era controlada pela observação; as crianças que necessitavam de estímulo eram puxadas pelos pés por uma faixa de tecido. Richard Pattle e Jonh Clemente's descobriram, em 1957, as propriedades da parede interna do alvéolo; em 1959, Mary Ellen Avery's e Jere Mead's descreveram a deficiência de surfactante como etiologia da HMD (síndrome respiratória) sendo esta a causa de 2500 mortes por ano, e desta forma proporcionou uma explicação para o distúrbio e uma base para a terapia (DOWNES, 1992; LUSSKY, 1999).

Ainda em 1957, na Cidade do CaboÁfrica do Sul, o pediatra Patrick Smythi e o anestesiologista Arthur Bull, cuidaram de recém-nascidos com tétano e ventilaram mecanicamente dez lactentes por até dez dias utilizando traqueostomia e bloqueio neuromuscular. Desta forma, eles comprovaram que os recém-nascidos poderiam ser ventilados mecanicamente com aparelhos de adultos modificados. Nesta década, Virgínia Apgar desenvolveu um método de contagem que avaliava as condições do recém-nato nos primeiros momentos de vida (DOWNES, 1992).

A partir da década de 70 os pediatras, cirurgiões e anestesiologistas infantis defenderam a idéia do agrupamento de lactentes com patologias severas em unidades especiais, visando o tratamento mais eficaz. As maternidades para prematuros passam a ser denominadas maternidade de tratamento especial, e desta para unidade de tratamento intensivo para recém-nascido (DOWNES, 1992; LUSSKY, 1999).

\section{- No Brasil:}

OLIVEIRA (1999), descreve como um marco na história da pediatria brasileira a inauguração, em 1882, da Policlínica Geral do Rio de Janeiro, que possuía um consultório infantil e realizava cursos sobre doenças das crianças, ministrados pelo médico Artur Moncorvo de Figueiredo. Este por sua vez, baseado em um estudo sobre a mortalidade infantil no Rio de Janeiro (na época capital do país), solicitou ao governo a criação de uma cadeira de Clínica de Moléstia de Criança nas faculdades de medicina do país, localizadas no Rio de Janeiro e Bahia.

No ano de 1899, o Instituto de Proteção e Assistência a Infância do Rio de Janeiro, foi criado pelo médico Arthur Moncorvo Filho, tendo seu funcionamento efetivo iniciado no ano de 1901. Este era destinado a assistência de crianças até 14 anos sem distinção de raça ou nacionalidade, desde a sua vida intrauterina. Como parte deste Instituto criou-se o Dispensário de Moncorvo, que foi instalado em 14 de julho do ano de 

recém-nascidos no exterior e no Brasil: perspectivas para o saber de enfermagem na neonatologia (1870-1903). Revista Eletrônica de Enfermagem, v. 06, n. 02, p. 286-291, 2004. Disponível em www.fen.ufg.br

1901, e a creche Senhor Alfredo Pinto, que somente foi instalada no ano de 1908, sendo considerada a primeira creche popular da cidade do Rio de janeiro, ela possuía 21 leitos e 2 incubadoras da marca Lyon para os débeis e prematuros (ANUÁRIO DO BRASIL, 1922).

A partir dos estudos dos médicos Jaime Silvado e Antonieta Morpurgo e reflexão destes sobre os notáveis resultados obtidos com as incubadoras no cuidado aos prematuros no exterior, o diretor do Dispensário Moncorvo recebeu uma proposta de criar um serviço de incubadoras nesta instituição que, em 1903, já possuía 2 incubadoras da marca Lyon (BARBOSA \& OLIVEIRA, 2002).

Para NOVAES (1990), os cuidados desenvolvidos para os recém-nascidos e, em especial, para os prematuros adicionados do desenvolvimento de cuidados pré-natais específicos foram os introdutores de uma assistência de qualidade e responsáveis pela redução da morbimortalidade peri e neonatal.

\section{CONSIDERAÇÕES FINAIS}

O ponto de partida para o desenvolvimento de cuidados especiais com as crianças foram as elevadas taxas de mortalidade infantil, principalmente com os recém-nascidos. A partir do século XIX, os médicos passaram a avaliar mais detalhadamente o recém-nascido. Baseados em estudos, pediatras e obstetras entre outros, estabeleceram regras de cuidados com as crianças e desenvolveram equipamentos, entre eles a incubadora. Desenvolvida na segunda metade do século XIX, a incubadora foi considerada um equipamento revolucionário no cuidado ao recém-nascido prematuro.

O obstetra Pierre Budin é considerado, na literatura, como o pai da Neonatologia, sendo responsável pelo desenvolvimento dos princípios e métodos que passaram a formar a base da medicina neonatal. Constata-se que a partir do século $X X$ os avanços médicos e tecnológicos propiciaram 0 aumento na qualidade da assistência neonatal

O Brasil sofreu influências dos países mais desenvolvidos, e no início do século $X X$ a assistência à criança recém-nascida iniciava sua organização baseada nos métodos estrangeiros. Vale ressaltar que os notáveis resultados obtidos com o uso das incubadoras no cuidado aos recém-nascidos prematuros em Paris propiciaram a importação de incubadoras no Brasil.

\section{REFERÊNCIAS BIBLIOGRÁFICAS}

ANUÁRIO DO BRASIL. Assistência pública e privada no Rio de Janeiro- História e Estatística. Comemoração do Centenário da Independência Nacional. Rio de janeiro,1922. AVERY, G. B. Perspectivas em neonatologia. Neonatologia: Fisiopatologia e cuidado do recém-nascido.Rio de Janeiro: Artes Médicas,1978.

AVERY, G. B. Perspectivas na década de 1990. Neonatologia: Fisiopatologia e cuidado do recém-nascido Rio de Janeiro: Medsi, 1999.

BARBOSA, A. C.; OLIVEIRA, I. C. S.O Advento das incubadoras no exterior e no Brasil: um ensaio histórico. Pediatria Atual, Rio de janeiro, v.15, n.6, Jun, 2002.

DOWNES, J.J. Evolução histórica, estado atual e desenvolvimento prospectivo do tratamento intensivo pediátrico. Clínica de Terapia Intensiva, Rio de Janeiro, v.1, n.1, p. 1-25, 1992.

HARRISON, H. The premature baby book: a parent's guide to coping and caring in first years. New York: St. Martin's, 1946.

HESS, J. H. Chicago Plan for Care Premature Infantes. JAMA, v.146, n.10, p.891-893, July 7, 1951. Chicago. Disponível em : http://www.neonatology.org/classics/hess.chic agoplan.html. Acesso em 10/08/2002.

LUSSKY, R. C. A century of neonatal medicine. Minnesota Medical Association, Mineápolis, v.82, dec.1999.Disponível em: http://www.nmed.org/publications/MnMed199 9/December. Acesso em 08/06/2001. 
NOVAES, H. M. D. Processo de desenvolvimento tecnológico em saúde; demanda e uso de tecnologia-o consumo hospitalar de São Paulo. Departamento de Medicina Preventiva da Universidade de São Paulo, 1990.

OLIVEIRA, I. C. dos S. Da mãe substituta à enfermeira pediatra: a construção do saber da enfermagem à criança hospitalizada. Rio de Janeiro: Anna Nery, 1999.
SILVADO, J. Incubadoras. Revista Médica de São Paulo/ Jornal Prático de Medicina, Cirurgia e Higiene, São Paulo, v.6, n.7, p. 110, abr. 1903.

VIEIRA, M. do P. de A.et al. A pesquisa em história. São Paulo: Ática, 1989.

Texto recebido em 27/02/2004

Publicação aprovada em 30/04/2004 